\title{
Maternal immune activation and strain specific interactions in the development of autism-like behaviors in mice
}

\author{
JJ Schwartzer ${ }^{1,2}$, M Careaga $^{2,3}$, CE Onore ${ }^{2,3}$, JA Rushakoff ${ }^{4}$, RF Berman ${ }^{2,4}$ and P Ashwood ${ }^{2,3}$
}

It is becoming increasingly apparent that the causes of autism spectrum disorders (ASD) are due to both genetic and environmental factors. Animal studies provide important translational models for elucidating specific genetic or environmental factors that contribute to ASD-related behavioral deficits. For example, mouse research has demonstrated a link between maternal immune activation and the expression of ASD-like behaviors. Although these studies have provided insights into the potential causes of ASD, they are limited in their ability to model the important interactions between genetic variability and environmental insults. This is of particular concern given the broad spectrum of severity observed in the human population, suggesting that subpopulations may be more susceptible to the adverse effects of particular environmental insults. It is hypothesized that the severity of effects of maternal immune activation on ASD-like phenotypes is influenced by the genetic background in mice. To test this, pregnant dams of two inbred strains (that is, C57BL/6J and BTBR ${ }^{+}{ }^{t} \mathrm{t} / \mathrm{J}$ ) were exposed to the viral mimic polyinosinic-polycytidylic acid (polyl:C), and their offspring were tested for the presence and severity of ASD-like behaviors. To identify differences in immune system regulation, spleens were processed and measured for alterations in induced cytokine responses. Strain-treatment interactions were observed in social approach, ultrasonic vocalization, repetitive grooming and marble burying behaviors. Interestingly, persistent dysregulation of adaptive immune system function was only observed in BTBR mice. Data suggest that behavioral and immunological effects of maternal immune activation are straindependent in mice.

Translational Psychiatry (2013) 3, e240; doi:10.1038/tp.2013.16; published online 12 March 2013

\section{Introduction}

Autism Spectrum Disorders (ASD) are a class of neurodevelopmental disorders characterized by deficits in three domains including: impairments in social interactions, deficits in language and communication and increased stereotyped restrictive/repetitive behaviors and interests. ${ }^{1}$ Estimates of heritability among twin and sibling studies indicate a moderate genetic and a substantial environmental component to the etiology. ${ }^{2}$ Numerous reports have suggested that the maternal environment, particularly stressors that disrupt development in utero, has a significant role in the incidence of ASD. ${ }^{3-5}$ Of particular interest is the link between fetal gestation and the state of the maternal immune system activation during critical periods of development. Recent epidemiological reports suggest a strong association between periods of maternal immune activation, particularly during early pregnancy, and an increased risk of having a child with ASD. ${ }^{6,7}$ Although these reports suggest a relationship between maternal immune activation and ASD, they have not yet been able to demonstrate a causal link.

Mouse models allow for translational studies of genetic and environmental contributions to neurodevelopmental disorders that can potentially establish plausible etiologies in clinical populations. For example, numerous mouse models of maternal immune activation have demonstrated that maternal viral and bacterial infection alters offspring behavior ${ }^{8-10}$, resulting in ASD-like phenotypes. ${ }^{11}$ In addition, transgenic mutant mice and inbred strains have been examined to investigate possible genetic contributions to ASD-like behaviors. ${ }^{12-14}$ These studies led to the discovery of the BTBR $\mathrm{T}^{+}$ $\mathrm{tf} / \mathrm{J}$ (BTBR) mouse as an inbred strain predisposed to exhibit ASD-like behaviors compared with those of the speciestypical C57BL/6J (C57) mouse. ${ }^{15}$ These behaviors include reduced levels of reciprocal social interactions, ${ }^{16}$ a lack of species-typical sociability, ${ }^{15}$ unusual repertoire of ultrasonic vocalizations ${ }^{17}$ and significantly higher levels of repetitive selfgrooming. ${ }^{15}$

Although mouse models have successfully identified genetic and maternal environmental factors that contribute to ASD independently, few studies have considered how these two factors work in combination. It is important to fill this gap in our knowledge, given the recent emphasis on understanding the importance of gene $x$ environment interactions in human populations. ${ }^{18}$ Previous studies investigating the role of maternal immune activation on offspring development have only examined the effects on a single mouse strain, ${ }^{8,10,11,19}$

\footnotetext{
${ }^{1}$ Department of Psychiatry and Behavioral Sciences, University of California, Davis, CA, USA; ${ }^{2}$ The M.I.N.D. Institute, University of California, Davis, CA, USA; ${ }^{3}$ Department of Medical Microbiology and Immunology, University of California, Davis, CA, USA and ${ }^{4}$ Department of Neurological Surgery, University of California, Davis. One Shields Avenue, Davis, CA, USA

Correspondence: Dr JJ Schwartzer, Psychiatry and Behavioral Sciences, M.I.N.D. Institute, University of California, Davis, One Shields Avenue, Davis, CA 95618, USA. E-mail: jschwartzer@ucdavis.edu

Keywords: Polyl:C; maternal immune activation; BTBR; autism spectrum disorders; mouse; gene-environment

Received 29 November 2012; revised 12 January 2013; accepted 14 January 2013
} 
with few studies considering how genetic differences contribute to the behavioral phenotype. ${ }^{20,21}$ Moreover, while the BTBR mouse has been shown to exhibit behavioral patterns thought to be relevant to the core domains of ASD, ${ }^{15}$ it is unknown whether these behaviors can be further exacerbated by the effects of environmental insults, such as maternal immune system activation.

The BTBR mouse exhibits aberrant immune responses that may contribute to its characteristic phenotype. ${ }^{22}$ This link between immune system dysregulation and ASD-like behaviors parallels human clinical research reporting atypical immune system responses in individuals with ASD. ${ }^{23}$ For example, children with ASD have increased levels of several cytokines in the plasma, including interleukin (IL)-1 and IL-6. ${ }^{24}$ Moreover, production of the proinflammatory cytokine tumor necrosis factor (TNF) $\alpha$ is increased in response to in vitro stimulation of peripheral blood mononuclear cells from children with ASD, and this response is associated with increases in repetitive stereotypical behaviors. ${ }^{25}$ Given this link between immune system dysregulation and ASD, as well as the important role genetic makeup plays in susceptibility, it is important to take these factors into account when using animal models to elucidate the etiology of ASD. More specifically in mouse models, it is important to consider how the effects of environmental insults, such as maternal immune activation may be dependent on genetic variations across strains. To this end, it is hypothesized that maternal immune activation will further exacerbate ASD-like behaviors and immune dysregulation in a mouse model with pre-existing genetic susceptibility to the behavioral phenotype (that is, BTBR). To test this, C57 and BTBR dams were exposed to the viral mimic polyinosinic-polycytidiylic acid (polyl:C) to activate the immune system during gestation, and the offspring were tested for ASD-like behaviors. Following behavioral testing, splenocytes were removed and measured for differences in cytokine release following in vitro immune stimulation.

\section{Materials and methods}

Animals. Male and female C57 (Jackson Laboratory, Sacramento, CA, USA) and BTBR (Jackson Laboratory, Bar Harbor Maine) mice were bred and maintained by the Center for Laboratory Animal Research, at University of California, Davis, and maintained at ambient room temperature on a $12 \mathrm{~h}$ light/dark cycle (light on at 0600 hours). Additionally, 129/SvImJ mice (Jackson Laboratory, Sacramento, CA) were used as stimulus mice for the threechambered social approach task. Each strain was housed separately, and food and water were provided ad libitum. All behavioral procedures were performed during the first $4 \mathrm{~h}$ of the light cycle, and all procedures were performed with approval by the University of California, Davis Institutional Animal Care and Use Committee and in accordance with the guidelines provided by the National Institutes of Health Guide for the Care and Use of Laboratory Animals.

Maternal immune activation. Mice were mated overnight and females were checked daily for the presence of seminal plugs, noted as gestational day 0.5 (G0.5). On G12.5, pregnant female mice were weighed and injected with a single dose $(20 \mathrm{mg} / \mathrm{kg}$; i.p.) of polyl:C (Sigma Aldrich; St Louis, MO, USA) or saline vehicle as previously described. ${ }^{10}$ Each dam was returned to its cage and left undisturbed until the birth of its litter. All mice pups remained with the mother until weaning on postnatal day 21 (P21), at which time mice were group housed 3-4 per cage with same-sex littermates. One male and one female from each litter were used for all behavioral tests to reduce the likelihood of litter effects. There were a total of four possible conditions: C57-saline ( $n=16)$, C57-polyl:C $(n=18)$, BTBR-saline $(n=12)$ and BTBR-polyl:C $(n=12)$.

Ultrasonic vocalizations. On postnatal day 6 , mice were removed from the nest and placed in a $7.5 \mathrm{~cm}$ diameter holding cup in a sound-attenuated chamber. Ultrasonic vocalizations were recorded using a mini-3 bat detector tuned to $40 \mathrm{kHz}$ and Ultravox software (Noldus Information Technology, Leesburg, VA, USA) set to detect ultrasonic vocalizations with durations greater than $10 \mathrm{~ms}$ as previously described. ${ }^{26,27}$ An amplitude filter was used to eliminate extraneous peripheral noise. Only one male and female pup were tested from each litter, and pups were marked with nontoxic ink on their paws for identification and repeated recordings were taken on $\mathrm{P} 6, \mathrm{P} 8, \mathrm{P} 10, \mathrm{P} 12$ and $\mathrm{P} 14$.

Three-chamber social approach. Ten-week-old mice were tested for social preference using the automated threechambered social approach apparatus. ${ }^{28,29}$ Briefly, experimental mice were placed in the center chamber and allowed to habituate for $10 \mathrm{~min}$ followed by an additional $10 \mathrm{~min}$ of free exploration of all three chambers to confirm the absence of a side preference. Then, mice were returned to the center chamber while a novel 129/SvImJ mouse was placed under an inverted wire cup in one side chamber, and an identical empty wire cup was placed on the other side. Experimental mice were then given an additional $10 \mathrm{~min}$ to explore both chambers and measured for the time spent in each chamber. The chamber containing the novel mouse was counterbalanced to control for any bias in chamber preference. Experimental mice were video recorded during the social approach phase and later scored for the time they spent sniffing the novel mouse by two independent investigators blind to the treatments. A sociability score was calculated as the time in social chamber minus time in novel object chamber. All testing chambers were thoroughly cleaned with $70 \%$ ethanol in between each testing session.

Marble burying. One week following the social approach task, mice were habituated to clean Plexiglas cages $(37 \times$ $14 \times 12.5 \mathrm{~cm}$ ) filled with a $4 \mathrm{~cm}$ thick layer of clean corncob bedding for $10 \mathrm{~min}$. Following habituation, animals were returned to their home cage and 15 glass marbles were laid out in five rows of three marbles placed equidistance apart. Mice were then returned to the cages and allowed to explore under dim illumination for $10 \mathrm{~min}$. At the end of the $10 \mathrm{~min}$ period, animals were gently removed from the testing cages and the number of marbles buried was recorded. Only marbles covered by $75 \%$ or more bedding were counted as buried, as previously described. ${ }^{11}$ 
Grooming. Two days after marble burying, mice were observed for frequency of grooming behavior. Preliminary studies determined a significant alteration in grooming duration when bedding was placed in the cages. Therefore, all subsequent grooming measurements were made while mice were in an empty clean Plexiglas cage. Grooming times were analyzed as percent change from saline exposed C57 controls. Briefly, after $10 \mathrm{~min}$ of habituation, animals were video recorded for an additional $10 \mathrm{~min}$ and later scored for self-grooming behavior by two individuals blind to treatment conditions. Grooming was defined as time spent licking paws, washing the nose, face or scratching fur with any foot.

Cell stimulation. One week following behavior testing, mice were killed by cervical dislocation and spleens collected for tissue processing. Briefly, fresh spleens were homogenized using a dounce homogenizer to separate splenocytes from connective tissues and to generate cells in suspension. Cells were then pelleted and red blood cells lysed using ammonium chloride potassium lysis buffer. Cells $(5 \times$ $10^{6} \mathrm{ml}^{-1}$ ) were stimulated for $24 \mathrm{~h}$ in a complete Roswell Park Memorial Institute medium 1640 (Invitrogen, Carlsbad, CA, USA) media supplemented with $10 \%$ low endotoxin, heat inactivated fetal bovine serum (Invitrogen), $100 \mathrm{lU} \mathrm{ml} \mathrm{ml}^{-1}$ penicillin, and $100 \mathrm{IU} \mathrm{ml}{ }^{-1}$ streptomycin (Sigma, St Louis, MO, USA), $25 \mathrm{\mu g} \mathrm{ml}^{-1}$ gentimycin (Sigma), $50 \mu \mathrm{m}$ 2-mecaptoethanol (Sigma) with media alone or media containing phorbol myristate acetate (PMA)/ionomycin (100 $\mathrm{ng} \mathrm{ml}^{-1}$ / $100 \mathrm{um})$. After $24 \mathrm{~h}$, supernatants were collect and stored at $-80^{\circ} \mathrm{C}$.

Cytokine analysis. The quantification of IL-6, IL-10, IL-17 and $\mathrm{TNF} \alpha$ in supernatants was carried out using murine multiplexing bead immunoassays (Millipore, Billerica, MA, USA). Cytokine analysis was performed as recommended by the manufacturer's specifications. Briefly, $25 \mu$ of supernatant was incubated with antibody-coupled beads. After a series of washes, a biotinylated detection antibody was added to the beads, and the reaction mixture was detected by the addition of streptavidin-phycoerythrin. The bead sets were analyzed using a flow-based Luminex 100 suspension array system (Bio-Plex 200; Bio-Rad Laboratories, Hercules, CA, USA). Unknown sample cytokine concentrations were calculated by Bio-Plex Manager software using a standard curve derived from the known reference cytokine concentrations supplied by the manufacturer. A five-parameter model was used to calculate final concentrations and values are expressed in $\mathrm{pg} \mathrm{ml}^{-1}$. The sensitivity of this assay allowed the detection of cytokine concentrations with the following limit of detection: IL-6 (1.7 $\left.\mathrm{pg} \mathrm{ml}^{-1}\right), \mathrm{IL}-10\left(4.0 \mathrm{pg} \mathrm{ml}^{-1}\right), \mathrm{IL}-17\left(0.4 \mathrm{pg} \mathrm{ml}^{-1}\right)$ and $\operatorname{TNF} \alpha\left(0.7 \mathrm{pg} \mathrm{ml}^{-1}\right)$. Concentrations obtained below the limit of detection were assigned a value of limit of detection/2 for statistical comparisons. Supernatant aliquots had not undergone any previous freeze/thaw cycle.

Statistical analysis. Statistical analyses were carried out using SPSS version 20 (Chicago, IL, USA). For ultrasonic vocalizations, data were analyzed using repeated measures analysis of variance with strain, treatment and sex as between-subjects factors and postnatal day as withinsubjects factor. For social sniff time, grooming, and marble burying tasks, three-way analysis of variance (strain $\times$ treatment $\times$ sex) was performed followed by least-square difference post hoc analysis when appropriate. For social approach data, initial chamber preference and the presence of a social preference were determined by separate pairedsamples Student's t-test for each strain-treatment group. Cytokine data were log transformed to allow for parametric analysis and analyzed by two-way analysis of variance (strain $\times$ treatment). Within-strain differences were analyzed using a priori planned comparisons. Cytokine concentrations were correlated with sociability scores, grooming and percent of marbles buried using spearman's rho (see Supplementary Material). All statistical tests were two-tailed with the alpha set at 0.05 .

\section{Results}

Ultrasonic vocalizations. The number of calls elicited by pups differed across postnatal days $\left(F_{(4,53)}=47.62\right.$, $P<0.001)$ with fewer calls observed at older ages as shown in Figure 1a. There was a significant main effect for mouse strain, with BTBR offspring emitting more calls than C57 offspring $\left(F_{(1,56)}=29.37, \quad P<0.001\right)$. There was also a significant day $\times$ treatment interaction $\left(F_{(4,53)}=2.77\right.$, $P<0.05)$. Further analysis showed significant treatment effects on P8 $\left(F_{(1,56)}=4.54, P<0.05\right)$ and on P10 $\left(F_{(1,56)}\right.$ 9.28, $P<0.01)$. Individual group comparisons revealed that on P8 offspring of polyl:C-treated BTBR dams showed significantly increased numbers of ultrasonic vocalizations compared with BTBR offspring of saline-treated dams $(P<0.05)$. This effect of polyl:C was not seen in offspring of C57 dams $(P=0.60)$ (Figure 1b). By $P 10$, both C57 $(P<0.05)$ and BTBR $(P<0.05)$ offspring whose dams were exposed to polyl:C during gestation displayed increased number of calls compared with offspring of saline-treated dams of either strain (Figure 1c). Interestingly, on P10 C57 mice of dams exposed to polyl:C elicited as many calls in a 2 min period as offspring of BTBR mice exposed to saline (59 \pm 8.26 and $66 \pm 9.88$, respectively). These data depict a stepwise gene-environment interaction, in which strain or treatment independently increase the number of ultrasonic vocalizations but also have additive effects on pup-elicited calls. No significant sex differences were observed across any developmental time point $\left(F_{(1,56)}=0.07, P=0.79\right)$.

Social approach. During the initial habituation phase of the social approach task there were no preferences for either empty side chamber (chamber 1 versus chamber 3 ) across either strain or treatment groups (Figure 2a). Although the BTBR offspring of polyl:C-exposed dams appeared to show a slight preference for chamber three, this preference was not statistically significant $(t(13)=1.63, P=0.129)$. As shown in Figure 2b, C57 mice whose dams were exposed to saline during gestation showed a strong preference for the chamber housing a novel mouse $(t(14)=6.03, P<0.001)$. In contrast, C57 offspring born from polyl:C-exposed dams displayed decreased preference for the chamber containing the novel mouse. However the preference for the novel mouse 


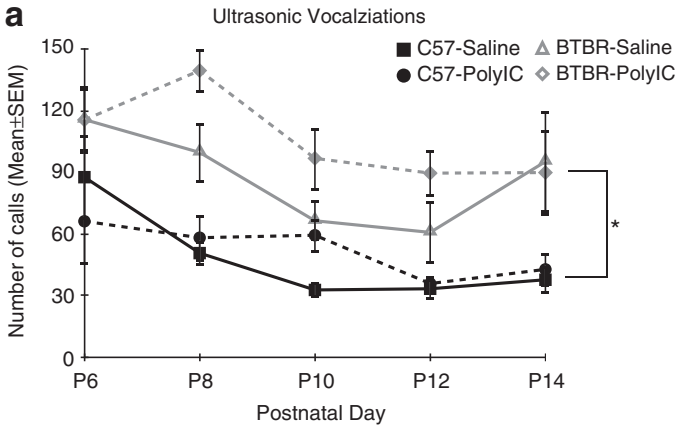

b

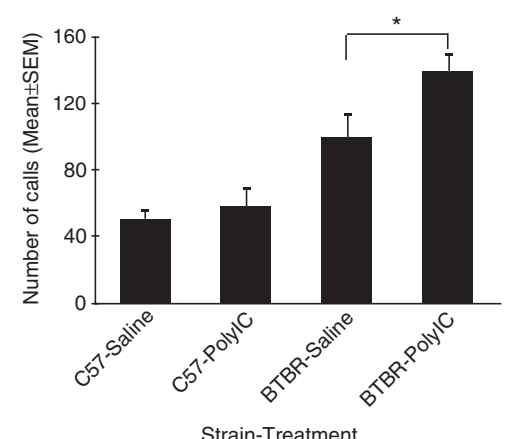

C

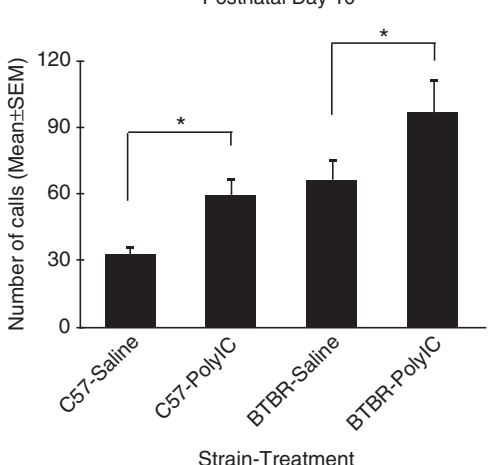

Figure 1 Number of calls elicited during pup separation. (a) BTBR pups elicited more calls than $C 57$ mice throughout postnatal development $\left({ }^{*} P<0.05\right)$. (b) On postnatal day 8, only BTBR offspring of polyl:C-treated dams produced more calls than offspring of saline-treated dams $\left({ }^{*} P<0.05\right)$. (c) On postnatal day 10 , both C57 and BTBR mice exposed to polyl:C during gestation produced a significantly greater number of calls compared with saline-exposed controls of the same strain $\left({ }^{\star} P<0.05\right)$.
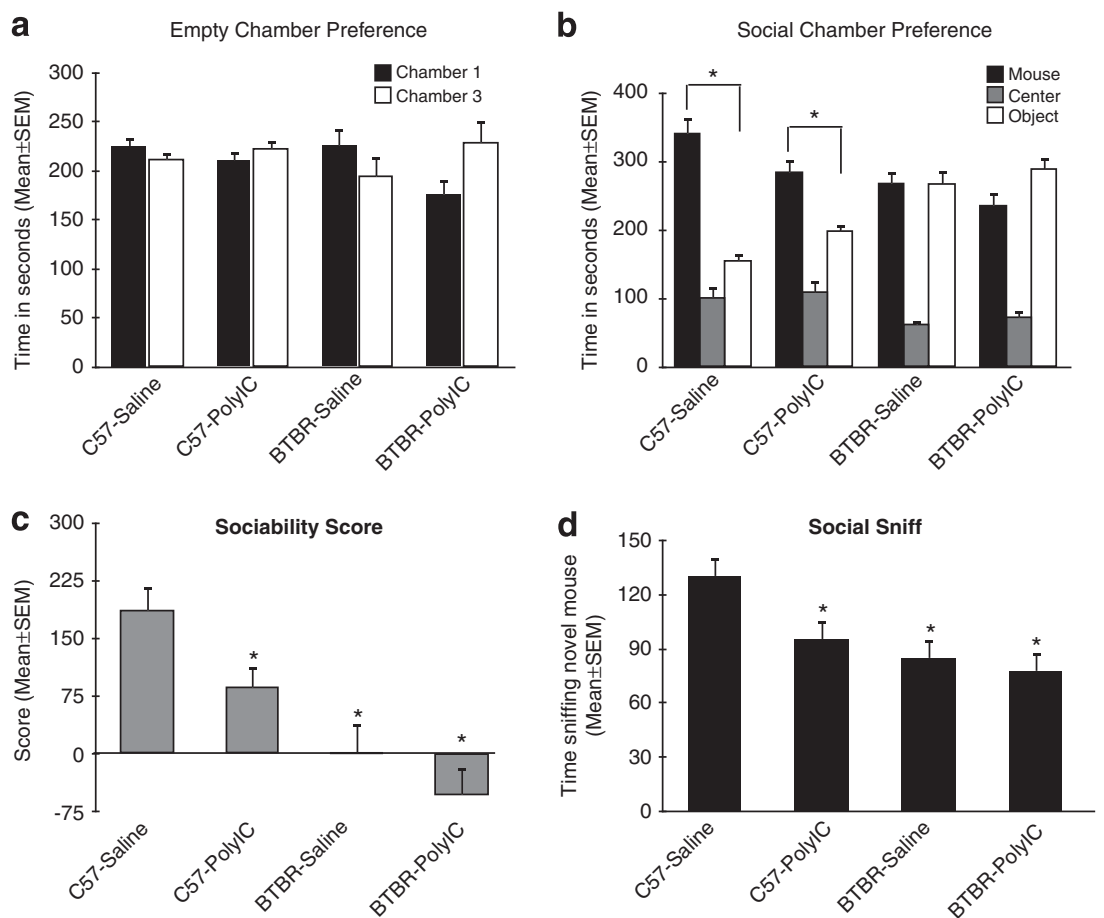

Figure 2 Maternal immune activation decreases social approach and social sniff. (a) No significant chamber preferences were found during the initial habituation period. (b) C57 offspring from saline and polyl:C-exposed dams showed significant preferences for the chamber with the novel mouse ( $\left.{ }^{\star} P<0.05\right)$, although the magnitude of preference was lower in the C57-polyl:C group. BTBR offspring from both saline and polyl:C-treated dams failed to show a preference for the chamber with the novel mouse. (C) Analysis of sociability scores showed that C57 offspring of polyl:C-treated dams were significantly less social than C57 mice from saline-treated dams ( ${ }^{*} P<0.05$ ). Although BTBR mice from both saline and polyl:C treatment dams had reduced sociability scores compared with C57-saline controls $\left({ }^{*} P<0.05\right)$, no differences were observed between BTBR treatment groups. (d) C57 offspring of polyl:C-exposed dams showed less social sniff time than C57 offspring of saline-exposed C57 dams ( $\left.{ }^{*} P<0.05\right)$. Both groups of BTBR offspring also showed less social sniff time than C57 offspring of saline-exposed C57 dams $\left({ }^{*} P<0.05\right)$. No significant differences were found in social sniff times between BTBR mice of either treatment condition.

chamber was still significantly greater than for the chamber containing the object $(t(17)=3.21, P<0.01)$. In contrast, BTBR offspring whose dams were exposed to saline failed to display a preference for the chamber with the novel mouse over the chamber with the object (that is, lack of preference for social stimuli) $(t(11)=0.02, P=0.99)$. A similar asocial behavior was observed in BTBR offspring of dams exposed to polyl:C $(t(11)=-1.47, P=0.17)$, (Figure $2 b)$.
The sociability score, a measure of the magnitude of preference for a social stimulus, was determined by the difference between the time spent in the chamber with a novel mouse minus the time spent in the chamber with a novel object. ${ }^{30} \mathrm{~A}$ three-way analysis of variance revealed significant strain $\left(F_{(1,56)}=22.63, P<0.001\right)$ and treatment $\left(F_{(1,56)}=\right.$ 5.20, $P<0.05)$ effects. The main effect for sex was not statistically significant $\left(F_{(1,56)}=0.01, P=0.91\right)$. Post hoc 
analysis revealed a significant decrease in the sociability score for C57 offspring from dams exposed to polyl:C compared with offspring of saline-treated C57 dams $(P<0.05)$ (Figure 2c). Interestingly, BTBR offspring from polyl:C-treated dams displayed significantly lower sociability scores compared with C57 offspring from both polyl:C $(P<0.01)$ and saline $(P<0.01)$-treated dams.

Finally, Figure 1d shows that the time spent social sniffing was significantly different between strains $\left(F_{(1,56)}=6.66\right.$, $P<0.05)$ and treatment groups $\left(F_{(1,56)}=4.25, P<0.05\right)$. These differences occurred independent of sex $\left(F_{(1,56)}=\right.$ $0.001, P>0.97)$. Post hoc analysis revealed that C57 offspring of dams exposed to polyl:C during development spent significantly less time sniffing the novel mouse compared with C57 offspring of saline exposed dams $(P<0.05)$. Similarly, both groups of BTBR offspring spent less time sniffing the novel mouse compared with $\mathrm{C} 57$ offspring of saline-treated dams $(P<0.05)$. No differences were observed between BTBR treatment groups.

Marble burying. During the $10 \mathrm{~min}$ marble burying task (Figure 3), offspring of BTBR dams buried a larger percent of marbles than offspring of C57 dams $\left(F_{(1,56)}=51.66\right.$, $P<0.001)$. In addition, offspring of dams exposed to polyl:C buried a larger percent of marbles than offspring of dams exposed to saline $\left(F_{(1,56)}=10.48, P<0.01\right)$. Within mouse strain comparisons showed that C57 offspring of dams exposed to polyl:C buried a higher percent of marbles than offspring of saline-exposed dams $(P<0.05)$. Despite this increase, C57 offspring of polyl:C-exposed dams buried fewer marbles compared with BTBR offspring of saline-exposed dams $(P<0.05)$. Finally, BTBR offspring of polyl:C-exposed dams buried a higher percentage of marbles compared with all other groups $(P<0.05$ for all comparisons) (Figure 3$)$.

Grooming. Time spent grooming for C57 offspring of salineexposed dams was used as a baseline (that is, 100\%) and percent change in grooming from this baseline was calculated for the other groups as shown in Figure 4. Statistical analysis showed a significant difference in grooming between

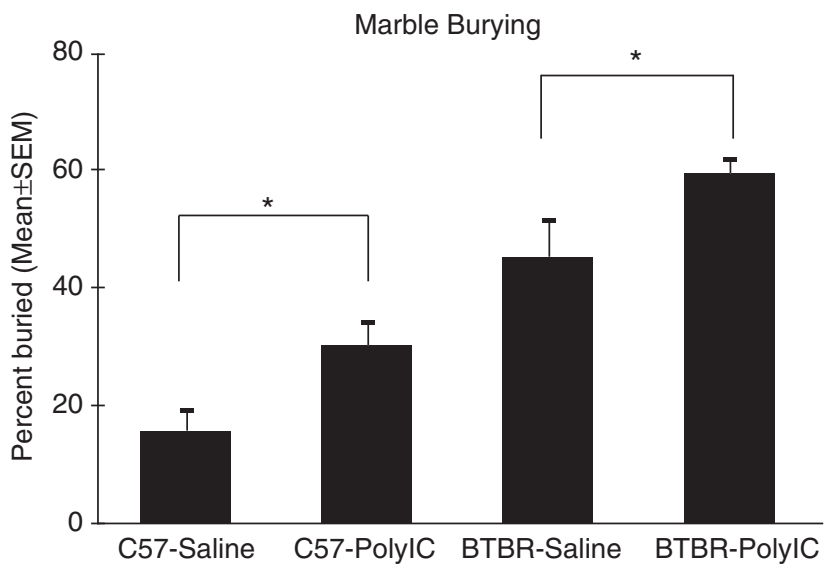

Figure 3 Marble burying behavior. BTBR offspring buried significantly more marbles than $\mathrm{C} 57$ offspring $(P<0.001)$. In addition, offspring of $\mathrm{C} 57$ and BTBR dams exposed to polyl:C buried more marbles than same strain offspring of dams exposed to saline ( $\left.{ }^{*} P<0.05\right)$. strains, with BTBR offspring showing more grooming than C57 offspring $\left(F_{(1,56)}=3.93, P<0.05\right)$. There was also a significant sex by treatment interaction $\left(F_{(1,56)}=5.17\right.$, $P<0.05)$, and therefore data for male and female offspring are presented separately in Figures $4 a$ and b, respectively. As shown in Figure 4a, male BTBR offspring of polyl:Cexposed dams showed significantly more grooming than male BTBR offspring of saline-exposed dams $(P<0.05)$. This effect of polyl:C exposure was not significant in male C57 offspring or in female BTBR or C57 offspring.

Dynamic immune response. Analysis of splenocyte responses to stimulation by phorbol myristate acetate/ ionomycin, revealed significant increases in immune response between mice strains (IL-6, $F_{(1,45)}=16.53$, $P<0.001$; IL-10, $F_{(1,45)}=20.60, P<0.001 ; \mathrm{IL}-17, F_{(1,45)}=$ 18.91, $P<0.001$; and TNF $\left.\alpha, F_{(1,45)}=18.91, P<0.001\right)$, with BTBR mice expressing increased concentrations of all cytokines measured (Figure 5). While maternal polyl:C exposure had no effect on IL-6 production in either strain (Figure 5a), a significant strain $\times$ treatment interaction was observed in the release of IL-17 $\left(F_{(1,45)}=5.44, P<0.05\right)$ (Figure $5 \mathrm{~b})$, and increases of IL-10 $\left(F_{(1,45)}=3.29, P=0.08\right)$ and of TNF $\alpha\left(F_{(1,45)}=3.16, P=0.08\right)$ approached statistical significance (Figures $5 \mathrm{c}$ and $\mathrm{d}$ ). Planned comparisons for polyl:C treatment effects within strain showed that offspring of polyl:C-treated BTBR dams produced greater levels of IL$17(t(22)=2.82, P<0.05)$ and IL-10 $(t(22)=2.02, P=0.05)$ compared with offspring of BTBR-saline controls, while the increase in TNF $\alpha$ was not significant $(t(22)=1.82, P=0.08)$. For C57 offspring, maternal exposure to polyl:C did not affect release of any of the four cytokines measured. Concentrations of all four cytokines measured were significantly correlated with marble burying and grooming behavior, but not sociability scores (see Supplementary Table S1). These associations were most apparent across strains (see Supplementary Figures S2-S4).

\section{Discussion}

Although heritability of ASD is substantial, the discordance rate in ASD among monozygotic twins is also large,,$^{2,31-35}$ making it apparent that genetic factors alone cannot account for the incidence of ASD. Instead, it is likely that an interaction between genetic predisposition and environmental insult during early prenatal or postnatal development contributes to the etiology of ASD. The current results document previously reported differences between C57 and BTBR mouse strains in several behaviors relevant to autism, including increased pup ultrasonic vocalizations, ${ }^{17}$ reduced social approach, and more self-grooming behaviors in the BTBR. ${ }^{15}$ In addition, the present results also show that a single polyl: $C$ injection on gestational day 12.5 can increase vocalizations, impair social behaviors and increase marble burying, with many of these effects being substantially more robust in BTBR mice. However, polyl:C-induced changes were not observed during the social approach task in BTBR mice. This observation is likely due to a floor effect as BTBR mice inherently lack social preference in the three-chambered social approach task. Interestingly, the behavioral deficits 
a

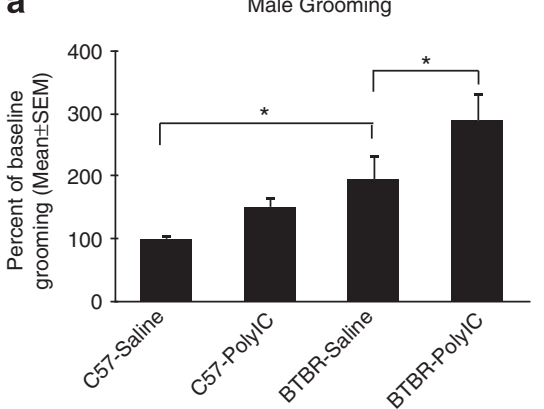

b

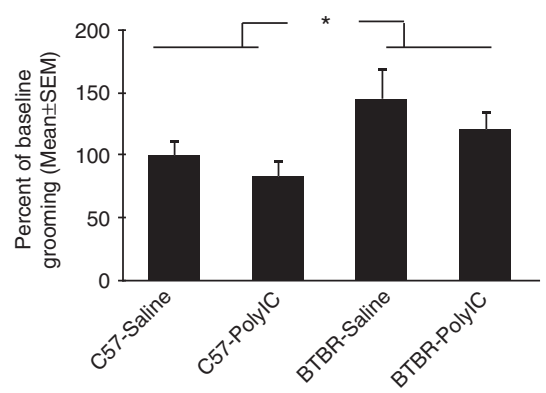

Figure 4 Maternal immune activation and sex-specific increases in grooming. (a) Overall, male BTBR offspring showed more grooming than male C57 offspring $(P<0.05)$. BTBR offspring of polyl:C-treated dams showed significantly more grooming than BTBR offspring from saline-treated dams. The apparent difference between treatment groups of C57 mice did not reach significance. (b) Overall, female BTBR offspring showed significantly more grooming than female C57 offspring $\left({ }^{\star} P<0.05\right)$. However, for females there were no significant treatment effects on grooming for either strain.
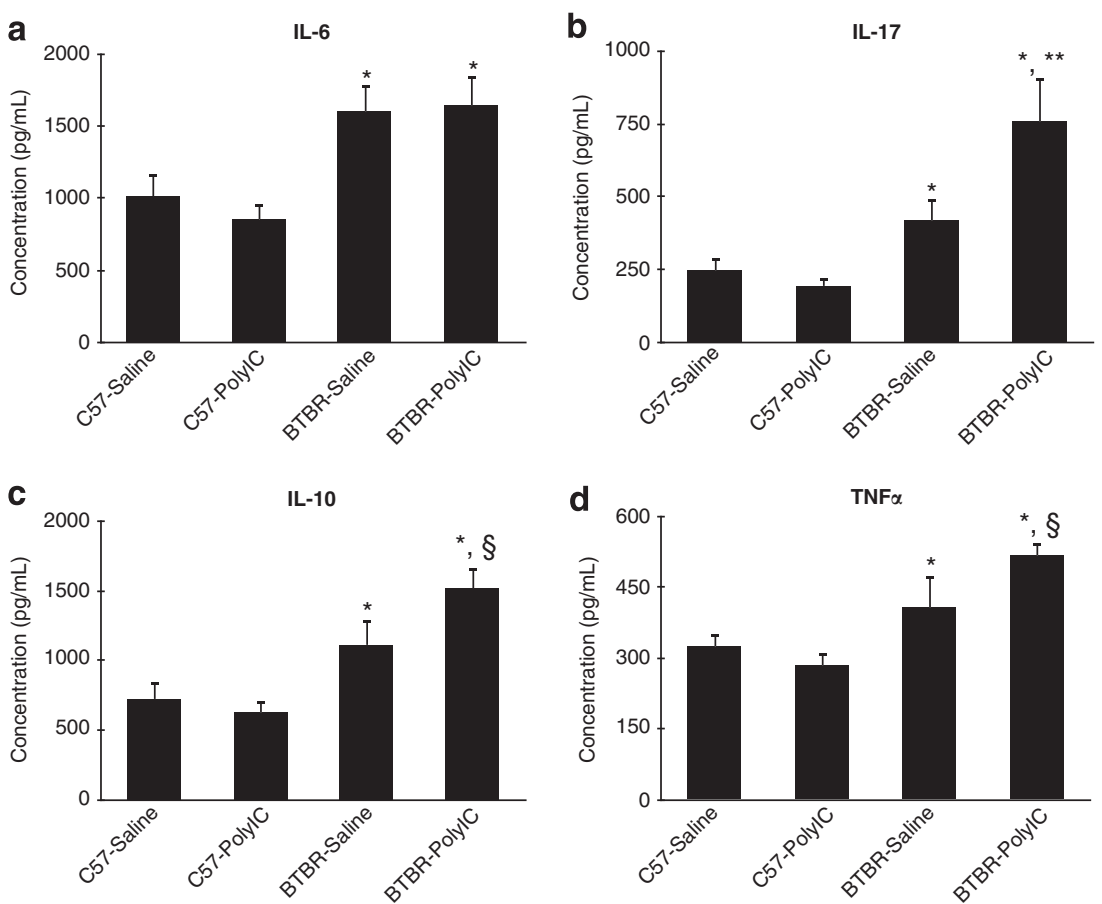

Figure 5 Dysregulated cytokine responses in offspring of polyl:C-treated dams. (a) IL-6 levels were significantly higher in BTBR than C57 offspring ( $\left.{ }^{\star} P<0.05\right)$. (b) IL-17 levels were significantly elevated in BTBR offspring versus $\mathrm{C} 57$ mice $\left({ }^{*} P<0.05\right)$ and levels were higher in BTBR-polyl:C offspring than BTBR-saline offspring $\left({ }^{* *} P<0.05\right)$. (c) BTBR mice expressed greater concentrations of IL-10 compared with C57 mice and increased levels of IL-10 in BTBR offspring of polyl:C-treated dams compared with BTBR mice of saline-exposed dams approached significance $\left({ }^{\S} P=0.05\right)$. (d) Both BTBR treatment groups expressed higher concentrations of TNF $\alpha$ compared with C57 mice with even greater increases observed in BTBR offspring of polyl:C-treated dams, though not statistically significance $\left({ }^{\S} P=0.08\right)$.

observed after maternal immune activation on C57 mice closely resembled the phenotype of typical BTBR mice (that is, BTBR-saline). These findings demonstrate that either genetic predisposition or environmental factors may have similar effects on behavior, but the combination of genetic predisposition (that is, BTBR mouse strain) and exposure to an environmental insult (that is, polyl:C) appears to be synergistic resulting in greater behavioral impairment than from either factor alone. Therefore, the complex spectrum of behavioral symptoms observed in human ASD populations may be due to the contributions of genetic, environment and to the additive effects of these factors.

Previous research in mouse models relevant to ASD has underscored the importance of both genetic makeup and the environment in the development of ASD-like behaviors. For example, systematic phenotyping of ASD-like behaviors across mouse strains has revealed a spectrum of behavioral responses dependent on genetic background. ${ }^{12,13,36}$ These studies identified the BTBR strain as a model organism that exhibits behavioral deficits modeling all three core domains of 
ASD. ${ }^{15}$ Similarly, studies have used mouse models to examine the effects of viral and bacterial infection on offspring behavior (for review see ${ }^{9}$ ). For example, Malkova et al. ${ }^{11}$ demonstrated a direct link between maternal polyl:C exposure and the expression of ASD-like behaviors in exposed offspring. Many of the behavioral outcomes observed in offspring of dams exposed to polyl: $\mathrm{C}$ in the current report are in line with previously reported findings, including deficits in social interaction and increased marble burying. However, increased grooming and more frequent ultrasonic vocalization calls are not in agreement. These difference may reflect variations in methodological approaches between reports including differences in the dose, developmental dates when injected, and the number of exposures to polyl:C. In addition, it is important to highlight the different approaches to mitigating litter effects between prior maternal immune activation studies and the current findings. One plausible explanation for discrepancies in the behavioral outcomes observed in the current study and prior reports may be due to the finding that maternal immune activation alters gene expression differently for each pup within a single litter. ${ }^{37}$ This notion would suggest that the effects of maternal polyl:C exposure on offspring behavior are dependent on additional factors and warrants further investigation into the mechanisms linking maternal immune activation to the expression of ASD-like behaviors.

Activation of the maternal immune system results in a cascade of inflammatory responses that are dependent on the pathogenic agent. The viral mimic polyl: $\mathrm{C}$ is a toll-like receptor 3 agonist that stimulates maternal immune responses resulting in behavioral alterations that are mediated through an IL- 6 dependent pathway. ${ }^{8}$ Blockade of IL- 6 activity by administering anti-IL- 6 antibodies inhibits the generation of social deficits in offspring of polyl:C-treated dams. ${ }^{10}$ Moreover, pregnant dams injected with IL-6 produce offspring exhibiting a phenotype similar to offspring of polyl:C-treated mothers, suggesting that polyl:C and IL-6 alter offspring behavior through a conserved signaling pathway. ${ }^{8}$ In both polyl:C and IL- 6 treatment regimens, IL- 6 activity mediates changes in hormone growth factors important for appropriate development. ${ }^{8}$ Moreover, maternal exposure to polyl:C alters synaptic protein expression and downregulates specific $\mathrm{N}$-Methyl-D-aspartate (NMDA) receptor subunits in the offspring ${ }^{38}$, suggesting a disruption in proper neuronal connectivity and an imbalance in excitatory/inhibitory inputs, ${ }^{39}$ two pathologies associated with ASD. ${ }^{40,41}$

The finding that a transient activation of the maternal immune system during gestation can produce lasting changes in offspring behavior underscores the importance of the prenatal environment on brain development and the emergence of complex behaviors. The gestational period is a time of critical development when perturbations can alter neuronal migration, differentiation and connectivity across the brain. ${ }^{42}$ Therefore, it is not surprising that environmental insults can produce differential effects on the brain depending on when the insult occurs during development. Indeed, maternal treatment with polyl:C has previously been shown to elicit distinct phenotypes in offspring dependent on whether the immune activating agent was given early or late in gestation. For example, offspring of dams exposed to polyl:C on gestational day 9 exhibit impaired sensorimotor gating, whereas mice from dams injected with polyl:C on gestational day 17 are characterized by impairments in working memory. ${ }^{19}$ BTBR and C57 mice in the current report were exposed to polyl:C on gestational day 12.5, a time point previously shown to result in autism-like behaviors in mouse offspring. ${ }^{10}$ These differences in behavioral phenotypes produced when the immune activating agent is introduced early, middle or late in gestation highlight the importance of critical developmental windows and demonstrate the diverse behavioral outcomes that can be produced with a single environmental insult.

In addition to the temporal onset of exposure to environmental agents, genetic makeup may also contribute to the expression of ASD-like behaviors and its underlying pathophysiology. In this study, we found that the effects of maternal immune activation were more pronounced in the BTBR strain when compared with the $\mathrm{C} 57$ strain, supporting the notion that the effects of maternal immune activation are dependent on pre-existing genetic factors. This finding warrants careful consideration of the genetic variations between strains that may give rise to their distinct phenotypes. To date, the genetic links to the BTBR's ASD-like behavioral profile are not wellunderstood and likely due to multiple genetic factors. One potential gene candidate that may contribute to the additive effects observed in the current report is the Disrupted In Schizophrenia 1 (Disc1) gene. BTBR mice are an inbred strain derived from the 129 mouse, ${ }^{43-45}$ a strain known to lack the Disc1 gene. ${ }^{46}$ Transgenic mice possessing a mutant form of Disc1 show increased anxiety, depression and social behavior deficits when born from dams exposed to polyl:C during gestation, ${ }^{47}$ demonstrating the important role that gene $\times$ environment interactions play in behavioral outputs. These Disc1 mutant mice were also reported to express altered cytokine levels that were futher disrupted when their dams were exposed to polyl:C. Similarly, BTBR mice in the current report produced greater release of IL-6, IL-17, IL-10 and $\mathrm{TNF} \alpha$ when challenged with phorbol myristate acetate/ ionomycin compared with C57 mice, and these increases were further exacerbated in offspring of dams whose immune system was activated during gestation by polyl:C. While our findings follow similar trends to previous reports, ${ }^{47}$ these similarities must be met with caution as the studies differ in the timing of polyl:C exposure. Interestingly, one report suggested that maternal immune activation is associated with the increase of IL-6 and IL-17 production in C57 offspring. ${ }^{48}$ However, in the current study, C57 offspring of polyl:C-treated dams tested as adults failed to exhibit alterations in IL-6 production, a finding consistent with other reports demonstrating altered cytokine levels in periadolescence but not adult offspring of maternal immune activated dams. ${ }^{49}$

The potential contributions of peripheral cytokine dysregulation on central nervous system function is an area of research that is gaining attention in the study of neurodevelopmental disorders. Cytokine receptors are expressed in the brain and exhibit region-specific difference in their distribution patterns across development. ${ }^{50,51}$ Although cytokine proteins do not readily penetrate the blood brain barrier, there are several mechanisms that are proposed to mediate cytokine signaling between the periphery and the brain. These pathways include active transport through the blood-brain 
barrier, ${ }^{52}$ activation of peripheral nerve fibers (for example, vagus nerve) that innervate cytokine releasing cells in the brain, ${ }^{53}$ and recruiting peripheral immune cells (that is, monocytes/macrophages) into the brain. ${ }^{54}$ Although cytokines were originally identified as immune-specific signaling molecules, it is becoming increasingly apparent that their release can modulate neuronal function. For example, IL-6 and TNF $\alpha$ modulate serotonin production through the activation of indoleamine-2,3-dioxygenase ${ }^{55}$, and TNF $\alpha$ release can alter both glutamate release and reuptake. ${ }^{56,57}$ In mice, disruption of cytokine signaling through receptor blockade or gene deletion produces cytokine-specific changes in ASDrelated behaviors, including memory deficits and social withdrawal (for review see ${ }^{23}$ ). Together, the complex interplay between immune and neural signaling along with our reported association between behavioral deficits and cytokine concentrations warrant future research on immune-related treatment strategies to target ASD-like behaviors.

In summary, our findings demonstrate the additive effects of genetic predisposition and maternal polyl:C exposure on the severity of ASD-like behaviors in mice. Moreover, maternal immune activation potentiates immune responses in offspring in a strain-dependent manner. These data further indicate the need to consider how genetic predisposition may exacerbate or protect against the effects of environmental insults in the etiology of ASD and underscore the importance of strain selection in the use of rodent models of neurodevelopmental disorders.

\section{Conflict of interest}

The authors declare no conflict of interest.

Acknowledgements. We thank Dr Brooke Babineau and Dr Jacqueline Crawley for their extensive training in the use of the social approach task, as well as their generosity in sharing their equipment. Additionally, we also thank Tamanna Noyon for her technical support. This work was supported by NIH T32MH073124, Jane Botsford Johnson Foundation, Peter Emch Foundation, Barbara and Michael Bass Foundation and the Brain and Behavior Research Foundation (formerly known as National Alliance for Research on Schizophrenia and Depression).

1. Association AP Diagnostic and Statistical Manual of Mental Disordrs. Revised 4th ed. Washington, DC, 2006.

2. Hallmayer J, Cleveland S, Torres A, Phillips J, Cohen B, Torigoe T et al. Genetic heritability and shared environmental factors among twin pairs with autism. Arch Gen Psychiatry 2011; 68: 1095-1102.

3. Newschaffer CJ, Croen LA, Daniels J, Giarelli E, Grether JK, Levy SE et al. The epidemiology of autism spectrum disorders. Annu Rev Public Health 2007; 28: 235-258.

4. Dufour-Rainfray D, Vourc'h P, Tourlet S, Guilloteau D, Chalon S, Andres CR. Fetal exposure to teratogens: evidence of genes involved in autism. Neurosci Biobehav Rev 2011; 35: 1254-1265.

5. Kinney DK, Munir KM, Crowley DJ, Miller AM. Prenatal stress and risk for autism. Neurosci Biobehav Rev 2008; 32: 1519-1532.

6. Atladottir HO, Thorsen P, Ostergaard L, Schendel DE, Lemcke S, Abdallah M et al. Maternal infection requiring hospitalization during pregnancy and autism spectrum disorders. J Autism Dev Disord 2010; 40: 1423-1430.

7. Brown AS. Epidemiologic studies of exposure to prenatal infection and risk of schizophrenia and autism. Dev Neurobiol 2012; 72: 1272-1276.

8. Hsiao EY, Patterson PH. Activation of the maternal immune system induces endocrine changes in the placenta via IL-6. Brain Behav Immun 2011; 25: 604-615.

9. Patterson PH. Immune involvement in schizophrenia and autism: etiology, pathology and animal models. Behav Brain Res 2009; 204: 313-321.

10. Smith SE, Li J, Garbett K, Mirnics K, Patterson PH. Maternal immune activation alters fetal brain development through interleukin-6. J Neurosci 2007; 27: 10695-10702.
11. Malkova NV, Yu CZ, Hsiao EY, Moore MJ, Patterson PH. Maternal immune activation yields offspring displaying mouse versions of the three core symptoms of autism. Brain Behav Immun 2012; 26: 607-616.

12. Moy SS, Nadler JJ, Young NB, Nonneman RJ, Segall SK, Andrade GM et al. Social approach and repetitive behavior in eleven inbred mouse strains. Behav Brain Res 2008; 191: 118-129.

13. Moy SS, Nadler JJ, Young NB, Perez A, Holloway LP, Barbaro RP et al. Mouse behavioral tasks relevant to autism: phenotypes of 10 inbred strains. Behav Brain Res 2007; 176: 4-20.

14. Roullet FI, Crawley JN. Mouse models of autism: testing hypotheses about molecular mechanisms. Curr Top Behav Neurosci. 2011; 7: 187-212.

15. McFarlane HG, Kusek GK, Yang M, Phoenix JL, Bolivar VJ, Crawley JN. Autism-like behavioral phenotypes in BTBR T + tf/J mice. Genes Brain Behav 2008; 7: 152-163.

16. Bolivar VJ, Walters SR, Phoenix JL. Assessing autism-like behavior in mice: variations in social interactions among inbred strains. Behav Brain Res 2007; 176: 21-26.

17. Scattoni ML, Gandhy SU, Ricceri L, Crawley JN. Unusual repertoire of vocalizations in the BTBR T +tf/J mouse model of autism. PLoS One 2008; 3: e3067.

18. Schwartzer JJ, Koenig CM, Berman RF. Using mouse models of autism spectrum disorders to study the neurotoxicology of gene-environment interactions. Neurotoxicol Teratol 2012; S0892-0362: 00148-1.

19. Meyer U, Nyffeler M, Yee BK, Knuesel I, Feldon J. Adult brain and behavioral pathological markers of prenatal immune challenge during early/middle and late fetal development in mice. Brain Behav Immun 2008; 22: 469-486.

20. Ehninger D, Sano Y, de Vries PJ, Dies K, Franz D, Geschwind DH et al. Gestational immune activation and Tsc2 haploinsufficiency cooperate to disrupt fetal survival and may perturb social behavior in adult mice. Mol Psychiatry 2012; 17: 62-70.

21. Ratnayake U, Quinn TA, Castillo-Melendez M, Dickinson H, Walker DW. Behaviour and hippocampus-specific changes in spiny mouse neonates after treatment of the mother with the viral-mimetic Poly I:C at mid-pregnancy. Brain Behav Immun 2012; 26: 1288-1299.

22. Heo Y, Zhang Y, Gao D, Miller VM, Lawrence DA. Aberrant immune responses in a mouse with behavioral disorders. PLoS One 2011; 6: e20912.

23. Onore $\mathrm{C}$, Careaga $\mathrm{M}$, Ashwood $\mathrm{P}$. The role of immune dysfunction in the pathophysiology of autism. Brain Behav Immun 2012; 26: 383-392.

24. Ashwood P, Krakowiak P, Hertz-Picciotto I, Hansen R, Pessah I, Van de Water J. Elevated plasma cytokines in autism spectrum disorders provide evidence of immune dysfunction and are associated with impaired behavioral outcome. Brain Behav Immun 2011; 25: 40-45.

25. Ashwood P, Krakowiak P, Hertz-Picciotto I, Hansen R, Pessah IN, Van de Water J. Altered T cell responses in children with autism. Brain Behav Immun 2011; 25: 840-849.

26. Braunschweig D, Golub MS, Koenig CM, Qi L, Pessah IN, Van de Water J et al. Maternal autism-associated IgG antibodies delay development and produce anxiety in a mouse gestational transfer model. J Neuroimmunol 2012; 252: 56-65.

27. Ta TA, Koenig CM, Golub MS, Pessah IN, Qi L, Aronov PA et al. Bioaccumulation and behavioral effects of $2,2^{\prime}, 4,4^{\prime}$-tetrabromodiphenyl ether (BDE-47) in perinatally exposed mice. Neurotoxicol Teratol 2011; 33: 393-404.

28. Yang M, Silverman JL, Crawley JN. Automated three-chambered social approach task for mice. Current protocols in neuroscience / editorial board, Jacqueline N Crawley [et al] 2011; Chapter 8(Unit 8): 26.

29. Nadler JJ, Moy SS, Dold G, Trang D, Simmons N, Perez A et al. Automated apparatus for quantitation of social approach behaviors in mice. Genes Brain Behav 2004; 3 : 303-314.

30. DeVito LM, Konigsberg R, Lykken C, Sauvage M, Young WS 3rd, Eichenbaum H. Vasopressin 1b receptor knock-out impairs memory for temporal order. J Neurosci 2009; 29: 2676-2683.

31. Folstein S, Rutter M. Infantile autism: a genetic study of 21 twin pairs. J Child Psychol Psychiatry 1977; 18: 297-321.

32. Folstein SE, Rosen-Sheidley B. Genetics of autism: complex aetiology for a heterogeneous disorder. Nat Rev Genet 2001; 2: 943-955.

33. Steffenburg S, Gillberg C, Hellgren L, Andersson L, Gillberg IC, Jakobsson G et al. A twin study of autism in Denmark, Finland, Iceland, Norway and Sweden. J Child Psychol Psychiatry 1989; 30: 405-416.

34. Ritvo ER, Spence MA, Freeman BJ, Mason-Brothers A, Mo A, Marazita ML. Evidence for autosomal recessive inheritance in 46 families with multiple incidences of autism. $A m \mathrm{~J}$ Psychiatry 1985; 142: 187-192.

35. Rosenberg RE, Law JK, Yenokyan G, McGready J, Kaufmann WE, Law PA. Characteristics and concordance of autism spectrum disorders among 277 twin pairs. Arch Pediatr Adolesc Med 2009; 163: 907-914.

36. Moy SS, Nadler JJ, Perez A, Barbaro RP, Johns JM, Magnuson TR et al. Sociability and preference for social novelty in five inbred strains: an approach to assess autistic-like behavior in mice. Genes Brain Behav 2004; 3: 287-302.

37. Garbett KA, Hsiao EY, Kalman S, Patterson PH, Mirnics K. Effects of maternal immune activation on gene expression patterns in the fetal brain. Translational psychiatry 2012; 2 : e98.

38. Forrest CM, Khalil OS, Pisar M, Smith RA, Darlington LG, Stone TW. Prenatal activation of Toll-like receptors-3 by administration of the viral mimetic poly $(\mathrm{l}: \mathrm{C})$ changes synaptic proteins, $\mathrm{N}$-methyl-D-aspartate receptors and neurogenesis markers in offspring. Mol Brain 2012; 5: 22. 
39. Wei H, Chadman KK, McCloskey DP, Sheikh AM, Malik M, Brown WT et al. Brain IL-6 elevation causes neuronal circuitry imbalances and mediates autism-like behaviors. Biochim Biophys Acta 2012; 1822: 831-842.

40. McPartland JC, Coffman M, Pelphrey KA. Recent advances in understanding the neural bases of autism spectrum disorder. Curr Opin Pediatr 2011; 23: 628-632.

41. Rubenstein JL, Merzenich MM. Model of autism: increased ratio of excitation/inhibition in key neural systems. Genes Brain Behav 2003; 2: 255-267.

42. Rice $D$, Barone $S$ Jr.. Critical periods of vulnerability for the developing nervous system evidence from humans and animal models. Environ Health Perspect 2000; 108(Suppl 3): 511-533.

43. Wahlsten D, Metten $P$, Crabbe JC. Survey of 21 inbred mouse strains in two laboratories reveals that BTBR $\mathrm{T} / \mathrm{t} \mathrm{t} / \mathrm{tf}$ has severely reduced hippocampal commissure and absent corpus callosum. Brain Res 2003; 971: 47-54.

44. Clee SM, Attie AD. The genetic landscape of type 2 diabetes in mice. Endocr Revi 2007; 28: $48-83$

45. Petkov PM, Ding Y, Cassell MA, Zhang W, Wagner G, Sargent EE et al. An efficient SNP system for mouse genome scanning and elucidating strain relationships. Genome Res 2004; 14: 1806-1811.

46. Clapcote SJ, Roder JC. Deletion polymorphism of Disc1 is common to all 129 mouse substrains: implications for gene-targeting studies of brain function. Genetics 2006; 173 2407-2410

47. Abazyan B, Nomura J, Kannan G, Ishizuka K, Tamashiro KL, Nucifora F et al. Prenatal interaction of mutant DISC1 and immune activation produces adult psychopathology. Biol Psychiatry 2010; 68: 1172-1181

48. Hsiao EY, McBride SW, Chow J, Mazmanian SK, Patterson PH. Modeling an autism risk factor in mice leads to permanent immune dysregulation. Proc Natl Acad Sci USA 2012; 109: 12776-12781.

49. Pacheco-Lopez G, Giovanoli S, Langhans W, Meyer U. Priming of Metabolic Dysfunctions by Prenatal Immune Activation in Mice: Relevance to Schizophrenia. Schizophr Bull 2011; sbr178v1-sbr178; PMID: 22186136.
50. Bauer S, Kerr BJ, Patterson PH. The neuropoietic cytokine family in development, plasticity, disease and injury. Nat Rev Neurosci 2007; 8: 221-232.

51. Garay PA, McAllister AK. Novel roles for immune molecules in neural development: implications for neurodevelopmental disorders. Front Synaptic Neurosci. 2010; 2 : 136

52. Banks WA, Kastin AJ, Durham DA. Bidirectional transport of interleukin-1 alpha across the blood-brain barrier. Brain Res Bull 1989; 23: 433-437.

53. Bluthe RM, Walter V, Parnet P, Laye S, Lestage J, Verrier D et al. Lipopolysaccharide induces sickness behaviour in rats by a vagal mediated mechanism. C R Acad Sci III 1994; 317: 499-503.

54. D'Mello C, Le T, Swain MG. Cerebral microglia recruit monocytes into the brain in response to tumor necrosis factoralpha signaling during peripheral organ inflammation. $J$ Neurosci 2009; 29: 2089-2102.

55. Pemberton LA, Kerr SJ, Smythe G, Brew BJ. Quinolinic acid production by macrophages stimulated with IFN-gamma, TNF-alpha, and IFN-alpha. J Interferon Cytokine Res 1997; 17: $589-595$

56. Ida T, Hara M, Nakamura $\mathrm{Y}$, Kozaki S, Tsunoda S, Ihara H. Cytokine-induced enhancement of calcium-dependent glutamate release from astrocytes mediated by nitric oxide. Neurosci Lett 2008; 432: 232-236.

57. Haroon E, Raison CL, Miller AH. Psychoneuroimmunology meets neuropsychopharmacology: translational implications of the impact of inflammation on behavior. Neuropsychopharmacology 2012; 37: 137-162.

(c) (i) $(\Theta)$ Translational Psychiatry is an open-access journal published by Nature Publishing Group. This work is licensed under the Creative Commons Attribution-NonCommercial-No Derivative Works 3.0 Unported License. To view a copy of this license, visit http://creativecommons.org/licenses/by-nc-nd/3.0/

Supplementary Information accompanies the paper on the Translational Psychiatry website (http://www.nature.com/tp) 